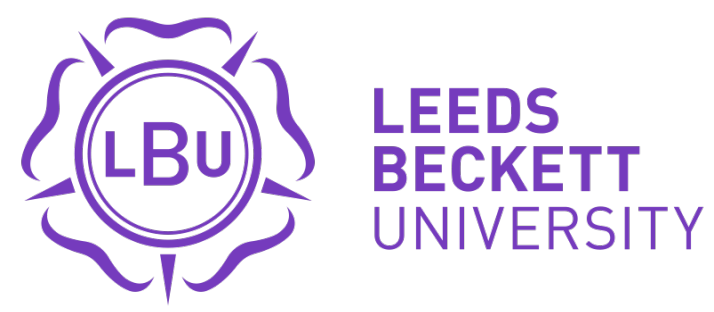

Citation:

Behringer, R and Ramachandran, M and Chang, V (2016) A Low-Cost Intelligent Car Break-In Alert System: Using smartphone accelerometers for detecting vehicle break-ins. In: The first International Conference on Internet of Things and Big Data, 23 - 25 Apr 2016, Rome, IT.

Link to Leeds Beckett Repository record:

https://eprints.leedsbeckett.ac.uk/id/eprint/2759/

Document Version:

Conference or Workshop Item (Updated Version)

The aim of the Leeds Beckett Repository is to provide open access to our research, as required by funder policies and permitted by publishers and copyright law.

The Leeds Beckett repository holds a wide range of publications, each of which has been checked for copyright and the relevant embargo period has been applied by the Research Services team.

We operate on a standard take-down policy. If you are the author or publisher of an output and you would like it removed from the repository, please contact us and we will investigate on a case-by-case basis.

Each thesis in the repository has been cleared where necessary by the author for third party copyright. If you would like a thesis to be removed from the repository or believe there is an issue with copyright, please contact us on openaccess@leedsbeckett.ac.uk and we will investigate on a case-by-case basis. 


\title{
A Low-Cost Intelligent Car Break-In Alert System Using Smartphone Accelerometers for Detecting Vehicle Break-Ins
}

\author{
Reinhold Behringer, Muthu Ramachandran, Victor Chang \\ School of Computing, Creative Technologies and Engineering, Faculty of Arts, Environment and Technology, Leeds Beckett \\ University, Leeds, LS6 3QS, United Kingdom \\ \{r.behringer,m.ramachandran,v.i.chang\}@leedsbeckett.ac.uk
}

Keywords: Alert system, smartphone, car alert, status detection, remote notification.

\begin{abstract}
Smartphones provide sensors and online data connectivity which makes them suitable for individual alert systems. A prototype for a car break-in alert system has been developed which can detect activity through smartphone accelerometers. The main goal of this system is to provide a low-cost alternative to expensive embedded systems with similar functionality. It can be controlled remotely solely through text messages from another mobile phone, which provides the option to use SMS instead of internet data access, in case of high cost of internet data connection (international roaming charges). In case of a detected break-in the system will send a text message to the user's second phone. The user also can query information and request the location of the vehicle. The prototype has been tested in various situations, and data have been collected to distinguish different scenarios. The system has been programmed with MIT App Inventor and will be made available for free on the Google Play Store.
\end{abstract}

\section{INTRODUCTION}

The rapid progress of smartphone technology often leads to users purchasing a new phone, even if the old phone is still working fine. A study by Entner (2011) has shown that in several countries users change their phone every two years on average. This raises the question what to do with the old phone, which may still be very capable for certain tasks, while the new phone would be the user's main phone for regular use.

One possibility is to find use of the old phone for a particular dedicated application, for example using the phone in a road vehicle, with the purpose of adding intelligent functionality to the car. In particular, if the old smartphone has a reasonable sensor suite (accelerometers, GPS), it can be used to detect motion and disturbance in the car, indicating a possible break-in into the vehicle by a burglar. Furthermore, the smartphone location system can be used to track and trace the vehicle when it is being stolen, and this allows a rapid response to such an incident. This opens the possiblilty for a very lowcost system which brings machine intelligence into a vehicle as a retro-fitted component: there is no additional hardware cost, and the cost for the software would be not more than for a typical smartphone app.

In this paper the prototype of such a system is described: Intelligent Car Break-In Alert System (ICaBrAS) consists of an "obsolete" smartphone that can be left in a vehicle and which can communicate with the user's main phone. The software for this invehicle phone has facilities to detect break-ins into the vehicle and removal of the vehicle from its parked location. The main communication link to the user is via text messaging, so no internet connection is required. If a mobile internet connection is available, it can be used for additional services such as using map services for determining location name.

Since Android is by far the most popular operating system with a market share of $82 \%$ in 2015 Q2 (IDC, 2015), it was chosen for the development of the ICaBrAS prototype. In order to include a wide range of Android versions and to simplify the development process, the MIT App Inventor prototyping tool (MIT, 2015) was used. This removed the hurdles of native Android programming and did allow quick concept testing without having to solve fundamental software development problems related to system interface or device/version compatibility. 
The "old" spare smartphone with the ICaBrAs sytem installed would need to be placed in the vehicle at a hidden location, but still with reasonable mobile and GPS satellite reception. The software for this alert system monitors the acceleration sensors and sends an alert message via SMS to the users "new" phone. The user then can remotely query the vehicle phone to obtain further information.

This provides a low-cost vehicle alert system without any fixed installation, wiring or other integration work at the vehicle itself. The prototype is self-contained and does not need any mechanical or electrical interface to the vehicle, except a connection to a power source, which in most cases would be the vehicle battery.

The alert system ICaBrAS will be made available on the Google Play Store for individual evaluation and testing.

\section{RELATED WORK}

\subsection{Vehicle Alert Systems}

There are numerous solutions for devices which set off a car alerts. High-end vehicles often have systems built-in which are directly linked with the vehicle data management systems. One example of such a system is the OnStar ${ }^{\circledR}$ system which can report vehicle diagnostics data to a repair centre or a mobile app (OnStar, 2015). There are subscription costs associated with this service.

There are also after-market systems available which can be retro-fitted into vehicles, for example from Maplin the Nikkai EasyFit Car Alarm (Maplin, 2015). This device sounds an alarm (120 dB) as soon as an electric consumer is switched on or a mechanical shock is "felt" by the sensor. This kind of alert is completely stand-alone and does not provide any link to the user's remote phone.

Recently there have been GPS trackers on the market, with GSM functionality for control through SMS messages. Most of these devices are embedded GPS receivers which only rely on location measurement to determine if the vehicle is being moved. These devices cost from $£ 50-£ 120$. There are, however, newer devices made in China for specifically addressing vehicle alerts, based on GPS and accelerometers. These devices are also linked to GSM with a SIM card and provide a functionality that is similar to ICaBrAS. They are, however not further configurable and lack the possibilities which a smartphone offers, for example automated call- back or customised audio responses. These devices are, however, very inexpensive at $£ 21$ (TomTop 2016).

\subsection{Sensing Acceleration}

The use of inexpensive smartphone sensors for invehicle application has been explored already since several years. Johnson and Trivedi (2011) have used accelerometers to determine the driving style. In general, such low-cost sensors are suitable for a wide variety of activity recognition applications, as shown by Saedi and El-Sheimy (2015).

Smartphone accelerometers have also been used in differentiating the transport medium in which the user is travelling (Bedogni, 2012), based on the characteristics of the acceleration data. Also, the sensors could detect a vehicle collision (Thompson et al, 2010).

The novelty in our current time in the year 2016 is that such a system can now be run on a low-cost "spare" smartphone. In this paper, there is also a new way of processing the smartphone data and detecting the specific characteristics by not examining the raw acceleration data, but by using the change of acceleration and acceleration oscillations (noise) to determine the kind of event that is being detected and classified.

\section{SYSTEM ARCHITECTURE}

The architecture of the alert system ICaBrAS is simple: once started, the app continuously captures data from the accelerometers and processes these data so that resulting values can be used to classify the detected motion activity. The data capture works as fast as the phone can capture and process the acceleration data. This means it draws significant battery current; therefore it is advisable to connect the power to the vehicle $12 \mathrm{~V}$ supply.
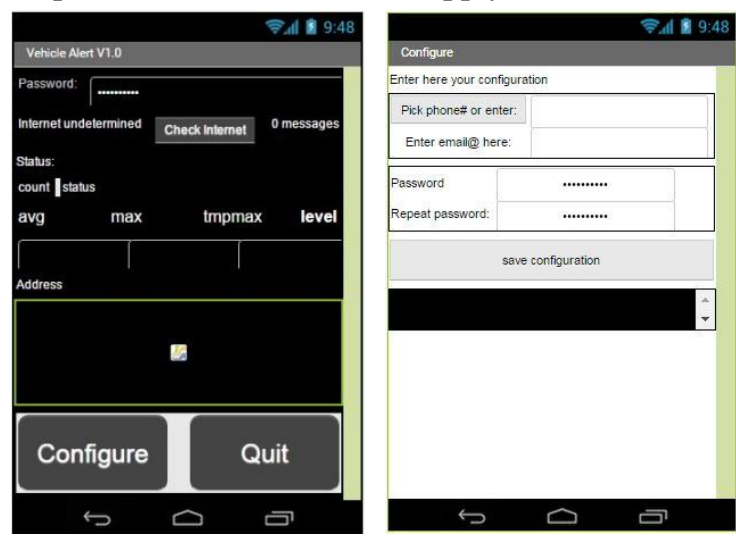

Figure 1. GUI of the application. Left: main operation screen (dark for stealthness). Right: configuration screen. 
The event-driven architecture of ICaBrAS responds to the following main events: (1) new accelerometer data are available, (2) new location data are available, and (3) new SMS has arrived. Callback functions handle these events and perform the necessary processing and actions.

\subsection{GUI on Smartphone}

The OS on this phone needs to be set to disable screen saver or going into sleep mode, because otherwise the acceleration data capture would stop. For a more advanced version other than the current prototype one would make the system run in the background as a continuous service and keep capturing the data and performing the processing.

ICaBrAS can be controlled either through a GUI on the phone screen (see Figure 1) or through SMS messages. The GUI shows status information and provides access to a configuration menu. Access to this configuration screen and also exit button are password-protected, so that no unauthorised user can configure or terminate the application while it is running. The background of the GUI is set to black, to preserve energy and to keep the phone screen dark while the application is running and the display is on, so that it cannot easily be seen by a possible intruder.

In the configuration menu the user can set the phone number of the corresponding second user phone from which ICaBrAS can be controlled. This allows only one single phone to control this app via SMS. Also the password can be set and changed in this configuration menu.

\subsection{Control through SMS}

The user can control the operation of ICaBrAS solely via SMS from a phone which has been "registered" with the software in the configuration screen. The configuration menu allows to either enter the phone number or select it from the address book on the phone. Incoming SMS messages from other phone numbers are ignored.

This method of using SMS for remote cotnrol has been chosen as a cost-effective method and also one that is widely compatible with all types of mobile phones. It works abroad and also in areas where the signal reception is not sufficient for mobile data access. Since only SMS messages are used, no data traffic is created. Such data traffic abroad could cost a significant amount of money, therefore the SMS control method is a way of keeping the operational costs down, as SMS messages are inexpensive even abroad.

If an SMS with a specific keyword is received, then ICaBrAS will respond with an SMS reporting the status and the activity classification of the system. Once remotely activated, ICaBrAS will keep sending SMS everytime a new (higher) activity level is detected. The user can then also stop this SMS reporting by sending a specific SMS.

The position of the vehicle can be requested through SMS: the returned SMS contains a link which will open Google Maps showing a map with the vehicle location.

The user can remotely request that $\mathrm{ICaBrAS}$ initiates a phone call back to the remote phone. In conjunction with a Bluetooth hands-free mic/speaker unit this allows to communicate with whoever is in the vehicle without them accessing or handling the phone.

\subsection{Development Environment}

For the development of this prototype, the cloudbased framework MIT App Inventor (MIT 2015) has been chosen. The reason is the robust and suitable functionality provided by this framework. The event-driven nature of the ICaBrAS prototype could easily be implemented with this framework, and there was no need to further consider compatibility issues between various versions of Android. This means that this prototype should be able to run on all mobile android phones from version 2.3 onwards which have a set of 3-axis accelerometer sensors and GPS for location reporting.

The development is done simply through dragand-drop in a graphical web-based programming environment. MIT App Inventor is intended to learn principles of mobile programming, but it extends very well to more demanding real-world applications like this.

\section{ACTIVITY CLASSIFICATION}

The main sensor for the activity detection on which the alert system is based is the set of accelerometers. Most smartphones that have been on the market in recent years have built-in 3-axis accelerometers. These sensors measure acceleration in the three orthogonal Cartesian coordinate axes. The acceleration data are used by applications to determine motion of the phone, for example to detect activity for sports and health applications (e.g. Hong et al, 2010), and to qualitatively determine key parameters of the motion. Hong et al. (2010) hereby 
use FFT to determine the motion characteristics to link and correlate it to calorie consumption. This method does not require bias removal, as only the changes of the acceleration values are taken into account.

For the purposes of this alert system, it is important to look not only at the temporal changes but also at the intensity of these changes, because they will lead to the classification of the detected activity. This triggers the need for dealing with one problem that all algorithms relying on accelerometer data face: the removal of the earth's gravity bias from the accelerometer data.

\subsection{Gravity Bias Removal}

Due to their nature, accelerometer data always do have a bias when being used in the presence of gravity: the gravity vector exerts a force which points directly to the centre of the Earth and which has the acceleration value of $1 \mathrm{~g}=9.81 \mathrm{~m} / \mathrm{s}^{2}$. This acceleration bias is present during all measurements of the overall acceleration $a$ which is calculated by the Euclidean Norm from the three Cartesian components:

$$
a=\sqrt{a_{x}^{2}+a_{y}^{2}+a_{z}^{2}}
$$

If the sensor is kept at a steady orientation, the bias removal is easy: the average value can be computed for each of the three components and is subtracted from each of the components in order to obtain the true acceleration. Since in this application the smartphone and its accelerometer sensors are placed in the vehicle at a steady location, this simple bias removal process can be implemented. It is to note, however, that this bias removal is not valid once the phone changes its orientation towards the earth, because then the gravity vector components change and the average value of the acceleration components will change. Such a situation can be mitigated by calculating a weighted or windowed average which only takes into account the most recent acceleration measurements.

In the ICaBrAS app the mean acceleration value of each of the three components is calculated through a low-pass filter with a time constant of 1 second. Each measurement $a_{i}$ leads to a low-pass filtered value $\overline{a_{\imath}}$ which represents the average over $\tau=1$ second. This value is subtracted from the measurement $a_{i}$ to provide the true acceleration without the gravity bias.

$$
\begin{gathered}
\overline{a_{\imath}}=k \cdot \overline{a_{\imath-1}}+(1-k) \cdot a_{i} \\
k=e^{-\frac{T}{\tau}}
\end{gathered}
$$

$$
a_{\text {true }}=a_{i}-\overline{a_{\imath}}
$$

This processing is triggered by the event when new accelerator data arrive. In the specific setup this was done at $50 \mathrm{~Hz}$, therefore the time period for the measurements was: $T=0.02 \mathrm{~s}$. It is explicitly measured every time the event is raised, therefore always the true value of the time period is taken into account in the computation of the true acceleration.

\subsection{Acceleration Processing}

In order to have a more convenient measure of the acceleration than the raw value which can range over several orders of magnitudes, the logarithm of the overall acceleration is calculated and processed through another low-pass filter with the same time constant $\tau=1$ second. This now provides two suitable values which can be used for describing motion and acceleration changes: the long-term filtered average baseline $\overline{\log a_{\text {true }}}$ value which describes the changes based on a time constant of 1 second and therefore can be used to detect general vehicle motion modes, and the immediate acceleration change value $\log a_{\text {true }}$ which describes sudden changes in acceleration and can therefore be used to detect sudden events which only last very short.

\subsection{Activity Classification}

The activity detection is based on these two values: $\log a_{\text {true }}$ and $\overline{\log a_{\text {true }}}$. The first value indicates peaks of activity, while the second value indicates a longer lasting commotion.

Table 1: Activity classification based on acceleration values.

\begin{tabular}{|c|c|l|c|}
\hline $\begin{array}{c}\text { maximum: } \\
\log \boldsymbol{a}_{\text {true }}\end{array}$ & $\frac{\text { average: }}{\log \boldsymbol{a}_{\text {true }}}$ & activity & level \\
\hline$<-3.3$ & $<-3.3$ & No activity. & 0 \\
\hline$-3.3<\mathrm{x}<-2.4$ & $<-3.3$ & Slight jerks. & 1 \\
\hline$-1.4<\mathrm{x}<-1.0$ & $<-3.3$ & Some jerks. & 2 \\
\hline$-1.0<\mathrm{x}<0.25$ & $<-3.3$ & $\begin{array}{l}\text { Significant } \\
\text { jerks. }\end{array}$ & 3 \\
\hline$>0.25$ & $<-3.3$ & Hard jerks. & 4 \\
\hline any & $-3.3<\mathrm{x}<-2.4$ & $\begin{array}{l}\text { Some } \\
\text { commotion. }\end{array}$ & 5 \\
\hline any & $-1.4<\mathrm{x}<-1.0$ & $\begin{array}{l}\text { Motor is } \\
\text { on. }\end{array}$ & 6 \\
\hline any & $-1.0<\mathrm{x}<0.25$ & $\begin{array}{l}\text { Vehicle is } \\
\text { driving }\end{array}$ & 7 \\
\hline any & $>0.25$ & $\begin{array}{l}\text { Phone in } \\
\text { user's hand. }\end{array}$ & 8 \\
\hline
\end{tabular}


Through heuristic evaluation the classification based on the acceleration values in Table 1 have been determined. These were obtained by observing the processed acceleration values in different situation. The mobile phone with its sensors was hereby placed on a flat surface in the vehicle while it recorded the acceleration data.

For determining the activity the log-acceleration values were observed during 2 -second intervals, and the peak (Column 1) and the average value (Column 2) of these log-acceleration values were recorded. They form the base for detecting a change in the activity level to which the phone is exposed. This activity is classified into one of 9 levels $(0-8$, in Column 4). For classifying a certain level, the conditions in both Column 1 and Column 2 need to be satisfied.

\section{EXPERIMENTAL SETUP}

The prototype of ICaBrAS was installed on a Google Nexus 4 mobile phone, running Android 5.0. The remote user phone which did receive SMS messages and was used to remotely control ICaBrAS is a Motorola Moto E with Android 5.0.2.

The ICaBrAS phone was placed in the rear of a 2005 Fiat Doblo 1.9 TD. It was continuously powered from the vehicle battery, and the OS was set to not go to sleep while connected to external power. The ICaBrAS software was started and kept running continuously for several days. The current that is drawn by the phone from the vehicle battery is around $300 \mathrm{~mA}$. Since the vehicle battery is a usual $12 \mathrm{~V}$ lead-acid with 90 Ah capacity, the theoretical operation duration can be 300 hours until the battery would be fully depleated. In typical realistic situations the usable battery capacity is only half the nominal value, which is $45 \mathrm{Ah}$. This leads to a real-world operation of around 150 hours. It is to note that when the vehicle is driven, the vehicle battery is being recharged again. To avoid draining the vehicle battery an auxiliary battery (leisure battery) as it is custom for camper vans can be used and can be connected to a solar panel. Provided the solar panel is rated at a sufficiently high power (150W is a realistic value for this), it then can generate continuous uninterrupted power to recharge the battery during the day even in winter.

The accelerometers of the Nexus 4 phone can run at $50 \mathrm{~Hz}$, therefore they provide 50 data sets per second. For debugging the ICaBrAS could be set into data recording mode, which provided sets of CSV files stored on an SDRAM card. This data recording was controlled remotely through SMS: a specific messages could switch on the data recording and turn it off, allowing to record the acceleration data in specific scenarios.

The phone was placed out of plain sight and was situated behind a fabric screen. This was done in order to avoid to attract attention by a possible intruder. The GPS reception still was good neough to provide location updates.

\section{RESULTS}

The Nexus 4 phone with the ICaBrAS software was run in a variety situations. The following graphical plots show the resulting log-acceleration data (maximum) in these sutiations.

In Figure 2 the log acc data are shown during no activity. This was recorded during idle time, and the data plot gives an impression of how the basic noise of the accelerometer data manifests itself in the case of absence of any motion or externally triggered jerk.

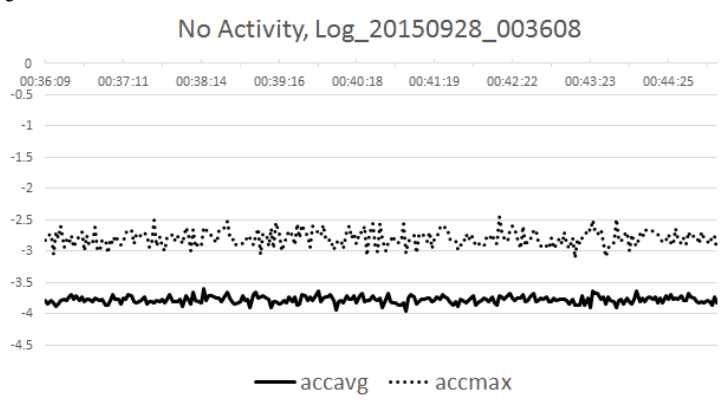

Figure 2. Acceleration during 9 minutes of no external activity. Peak (dotted) and average (solid) values are shown.

In Figure 3 the data plot shows the logacceleration values when the door is opened with a key. The peak value indicates the moment when the opening of the door occurs. This peak is detected and gives rise to a SMS being sent, indicating that an intruder has entered the vehicle.

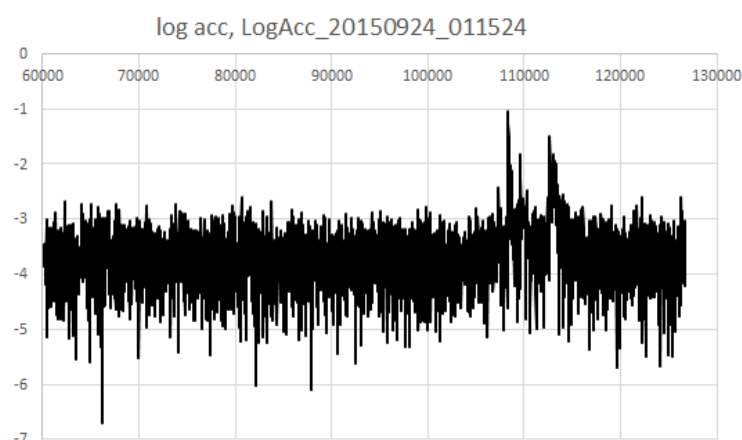

Figure 3. Raw log acceleration data with peak when opening the vehicle door. 
In Figure 4 the $\log$ acceleration is shown during several phases of driving. The first peak is when opening the door, then shutting it. At time 176,000 the motor is started, and then a constant acc noise level between -1 and -3 is recorded, which is clearly above the acc noise level for no motion. At time 191,000 the motor is switched off again, followed by some commotion which includes opening the door then shutting the door at 213,000 . The time units are milliseconds.

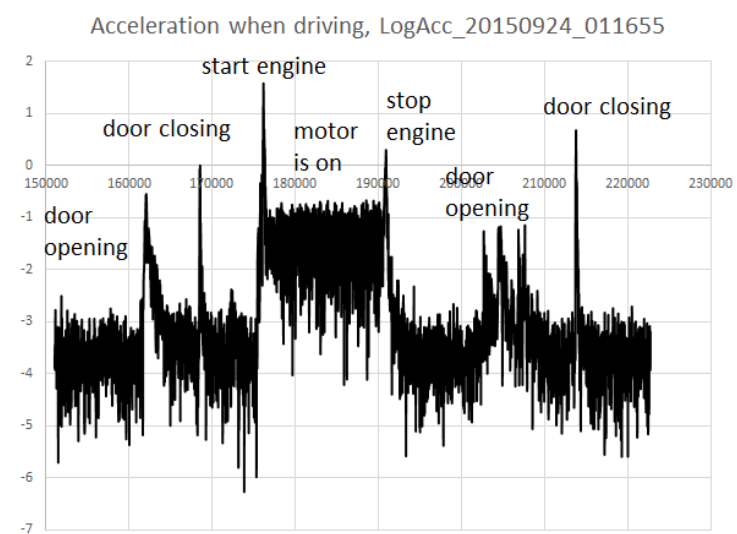

Figure 4. Log acceleration values during different phases of vehicle operation.

These data plots illustrate how the log acc data changed during different situation. The accelerometers in the smartphone are able to pick up differences in acceleration which can be used for determining and classifying the activity level which the phone and hereby the vehicle are undergoing.

The acceleration data which are being collected and processed while the vehicle is driving can also be used to identify road roughness. This can be used to highlight trouble spots with potholes and rough road surfaces, in particular if many vehicles are using this app and report back such data via crowdsourcing.

\section{CONCLUSIONS}

The experiments with this prototype app have indicated that it is possible to re-use an obsolete smartphone and use it as a low-cost vehicle alert system. Simple data processing is employed which can be run in real-time on a typical smartphone without exceeding its capabilities. This can provide a low-cost alternative to expensive on-board vehicle systems. With such a smartphone, any vehicle can be "upgraded" to become an intelligent device which can communicate its status and captured data. This can be used in the context of detecting a break-in, but also an actual theft of the vehicle, as this can report its current and changing location back to the user's "main" phone.

There is much further potential for using such an app for data harvesting: the app could collect data about road roughness and share it with other users through a web app / web portal. Also, in conjunction with the location tracking, the app could be used to estimate the mechanical stress under which the vehicle is, from its accelerations and vibrations which are recorded on the phone.

\section{REFERENCES}

Bedogni, L., Di Felice, M., Bononi, L. 2012. By train or by car? Detecting the user's motion type through smartphone sensors data. Wireless Days, 2012 IFIP, 21-23 Nov 2012, Dublin.

Entner, R, 2011. International Comparisons: The Handset Replacement Cycle. Recon Analysis. 23 June 2011. http://mobilefuture.org/wpcontent/uploads/2013/02/mobilefuture.publications.handset-replacement-cycle.pdf

Hong, Y.-J. and Kim, I.-J., 2010. Mobile health monitoring system based on activity recognition using accelerometer. In Simulation Modelling Practise and Theory, v.8, no.4, 4 April 2010, pp. 446-455.

IDC, 2015. Smartphone OS Market Share, 2015 Q2. http://www.idc.com/prodserv/smartphone-os-marketshare.jsp

Johnson, D.A. and Trivedi, M.M. 2011. Driving style recognition using a smartphone as a sensor platform. Proc of ITSC 2011, 14 ${ }^{\text {th }}$ Int. IEEE Conference on Intelligent Transportation Systems (ITSC). Washington DC, 5-7 Oct 2011, pp. 1609-1615.

Maplin, 2015. http://www.maplin.co.uk/p/nikkai-easy-fitcar-alarm-120db-siren-a78jq

MIT, 2015. MIT App Inventor. http://appinventor.mit.edu/explore/

OnStar, 2015. https://www.onstar.com

Saedi, S. and El-Sheimy, N. 2015. Activity Recognition Using Fusion of Low-Cost Sensors on a Smartphone for Mobile Navigation Application. Micromachines, vol 6, no.8, pp. 1100-1134.

Thompson, C,. White, J., Dougherty, B., Albright, A., and Schmidt, D.C. 2010. Using Smartphones to Detect Car Accidents and Provide Situational Awareness to Emergency Responders. In Mobile Wireless Middleware, Operating Systems and Applications. $3^{\text {rd }}$ Ing. Conf. Mobileware 2010, Chicago. Pp. 29-42.

TomTop, 2016. Vehicle Tracker Anti Theft Alert System, http://www.tomtop.com/various-function-gsm-gpscar-vehicle-tracker-anti-theft-alert-system-sos-voicetalking-communicating-iosandriod-app-positioningalarm-tracker-k2463.html 